\title{
Article
}

\section{Analysis of the qualitative behaviour of an eighth-order fractional difference equation}

\author{
Mohammed Bakheet Almatrafi ${ }^{1, *}$ and Marwa Mohammed Alzubaidi ${ }^{2}$ \\ 1 Department of Mathematics, Faculty of Science, Taibah University, P.O. Box 30002, Saudi Arabia. \\ 2 Department of Mathematics, College of Duba, University of Tabuk, P.O. Box 71491, Saudia Arabia.; \\ mmialzubaidi@hotmail.com. \\ * Correspondence: mmutrafi@taibahu.edu.sa
}

Received: 24 February 2019; Accepted: 04 April 2019; Published: 29 April 2019

\begin{abstract}
The exact solutions of most nonlinear difference equations cannot be obtained theoretically sometimes. Therefore, a massive number of researchers predict the long behaviour of most difference equations by investigating some qualitative behaviours of these equations from the governing equations. In this article, we aim to analyze the asymptotic stability, global stability, periodicity of the solution of an eighth-order difference equation. Moreover, a theoretical solution of a special case equation will be presented in this paper.
\end{abstract}

Keywords: Equilibrium, stability, boundedness, periodicity, qualitative behaviour.

MSC: 39A10.

\section{Introduction}

$\mathbf{M}$ ost fractional difference equations are studied from their qualitative behaviours of the governing equations. This can be strongly attributed to the fact that obtaining the exact solutions of these equations is quite sophisticated sometimes. A considerable number of authors has established the equilibria, stability, periodic solutions and boundedness of some nonlinear recursive relations. For example, Abdelrahman et al. [1] examined the asymptotic stability of the solutions of the following recursive equation

$$
x_{n+1}=a x_{n-l}+b x_{n-k}+f\left(x_{n-l}, x_{n-k}\right) .
$$

Almatrafi et al. [2] investigated the qualitative behaviour of the following fourth-order rational difference equations

$$
x_{n+1}=\frac{\alpha x_{n} x_{n-3}}{ \pm \beta x_{n-3} \pm \gamma x_{n-2}} .
$$

El-Moneam et al. [3] introduced some results on the qualitative behaviour of the following recursive equation

$$
x_{n+1}=A x_{n}+B x_{n-k}+C x_{n-l}+D x_{n-\sigma}+\frac{b x_{n-k}+h x_{n-l}}{d x_{n-k}+e x_{n-l}} .
$$

Garić-Demirović et al. [4] presented the periodic solution and the stability character of the following second-order relation

$$
x_{n+1}=\frac{A x_{n}^{2}+B x_{n} x_{n-1}+C x_{n-1}^{2}}{a x_{n}^{2}+b x_{n} x_{n-1}+c x_{n-1}^{2}} .
$$

In [5], the authors concerned with showing an analytical investigation about the following sixth-order difference equation

$$
x_{n+1}=\frac{C x_{n-5}}{A+B x_{n-2} x_{n-5}} .
$$


Moreover, Khaliq and Hassan [6] examined the dynamic behaviour of the recursive equation given on the form

$$
x_{n+1}=a x_{n}+\frac{\alpha+\beta x_{n-k}}{A+B x_{n-k}} .
$$

Elabbasy et al. [7] studied the periodic solution, local stability, global attractivity and the solution of a special case from the equation given by

$$
x_{n+1}=a x_{n}-\frac{b x_{n}}{c x_{n}-d x_{n-1}} .
$$

For more information about the dynamic behaviours of some fractional recursive equations, one can see [8-15].

Our main purpose in this paper is to investigate some qualitative behaviours such as the local stability, global stability and the periodicity of the following rational recursive equation

$$
x_{n+1}=c_{1} x_{n-3}+\frac{c_{2} x_{n-3}}{c_{3} x_{n-3}-c_{4} x_{n-7}}, \quad n=0,1, \ldots
$$

where the constants $c_{i}$, for all $i=1, \ldots, 4$, are assumed to be positive real numbers and the whole initial data are required to be arbitrarily real numbers. We also aim to present the exact solutions to a special case equation from Equation (1). Finally, numerical examples on each property will be included as well.

\section{Analysis of Local Stability}

In this section, we will examine the behaviour of the solutions around the equilibrium point and show that the solutions which start nearby the fixed point stay nearby the fixed point under a specific condition. The equilibrium point of Equation (1) is given by the following equation:

$$
\bar{x}=c_{1} \bar{x}+\frac{c_{2} \bar{x}}{c_{3} \bar{x}-c_{4} \bar{x}}
$$

which leads to

$$
\bar{x}=\frac{c_{2}}{\left(1-c_{1}\right)\left(c_{3}-c_{4}\right)}, c_{1} \neq 1, c_{3} \neq c_{4} .
$$

Suppose that a function $h:(0, \infty)^{2}-(0, \infty)$ is defined as following

$$
h(r, s)=c_{1} r+\frac{c_{2} r}{c_{3} r-c_{4} s} .
$$

Then,

$$
\begin{aligned}
& \frac{\partial h(r, s)}{\partial r}=c_{1}+\frac{c_{2}\left(c_{3} r-c_{4} s\right)-c_{2} c_{3} r}{\left(c_{3} r-c_{4} s\right)^{2}}=c_{1}-\frac{c_{2} c_{4} s}{\left(c_{3} r-c_{4} s\right)^{2}}, \\
& \frac{\partial h(r, s)}{\partial s}=\frac{c_{2} c_{4} r}{\left(c_{3} r-c_{4} s\right)^{2}} .
\end{aligned}
$$

Now, Equation (3) and Equation (4) will be evaluated at $\bar{x}$ as follows:

$$
\begin{aligned}
& \frac{\partial h(\bar{x}, \bar{x})}{\partial r}=c_{1}-\frac{c_{2} c_{4} \bar{x}}{\left(c_{3} \bar{x}-c_{4} \bar{x}\right)^{2}}=c_{1}-\frac{c_{4}\left(1-c_{1}\right)}{c_{3}-c_{4}}:=-p_{1}, \\
& \frac{\partial h(\bar{x}, \bar{x})}{\partial s}=\frac{c_{2} c_{4} \bar{x}}{\left(c_{3} \bar{x}-c_{4} \bar{x}\right)^{2}}=\frac{c_{4}\left(1-c_{1}\right)}{c_{3}-c_{4}}:=-p_{2} .
\end{aligned}
$$

Next, the liberalization of Equation (1) about the fixed point is given by the following form

$$
y_{n+1}+p_{1} y_{n-3}+p_{2} y_{n-7}=0 .
$$

Theorem 1. Let $\left|c_{1} c_{3}-c_{4}\right|+c_{4}\left|1-c_{1}\right|<\left|c_{3}-c_{4}\right|$. Then, the fixed point of Equation (1) is locally asymptotically stable. 
Proof. It can be simply observed from Theorem A of [16] that the equilibrium point of Equation (1) is locally asymptotically stable if

$$
\left|p_{1}\right|+\left|p_{2}\right|<1 \text {. }
$$

This gives us

$$
\left|-\left(c_{1}-\frac{c_{4}\left(1-c_{1}\right)}{c_{3}-c_{4}}\right)\right|+\left|-\frac{c_{4}\left(1-c_{1}\right)}{c_{3}-c_{4}}\right|<1,
$$

which can be written on the following form

$$
\left|c_{1}\left(c_{3}-c_{4}\right)-c_{4}\left(1-c_{1}\right)\right|+c_{4}\left|1-c_{1}\right|<\left|\left(c_{3}-c_{4}\right)\right| .
$$

Hence,

$$
\left|c_{1} c_{3}-c_{4}\right|+c_{4}\left|1-c_{1}\right|<\left|c_{3}-c_{4}\right|
$$

This complete the proof.

\section{Analysis of Global Stability}

We now turn to introduce an appropriate condition under which the fixed point of Equation (1) is a global attractor.

Theorem 2. Let $c_{1}<\frac{c_{2} c_{4} s}{\left(c_{3} r-c_{4} s\right)^{2}}$, then the equilibrium point of Equation (1) is a global attractor if $c_{1} c_{3}>c_{4}$.

Proof. Let $a, b \in \mathbb{R}$ and suppose that $h:[a, b]^{2}-[a, b]$ is a function defined by Equation (2). We assume that $c_{1}<\frac{c_{2} c_{4} s}{\left(c_{3} r-c_{4} s\right)^{2}}$, then the function $h$ is decreasing in $r$ and increasing in $s$. Now, we suppose that $(\phi, \psi)$ is a solution to the system given by

$$
\phi=h(\psi, \phi), \psi=h(\phi, \psi)
$$

Therefore,

$$
\begin{aligned}
& \phi=h(\psi, \phi)=c_{1} \psi+\frac{c_{2} \psi}{c_{3} \psi-c_{4} \phi} \\
& \psi=h(\phi, \psi)=c_{1} \phi+\frac{c_{2} \phi}{c_{3} \phi-c_{4} \psi} .
\end{aligned}
$$

Clearing the denominators gives

$$
\begin{aligned}
& c_{3} \phi \psi-c_{4} \phi^{2}=c_{1} c_{3} \psi^{2}-c_{1} c_{4} \phi \psi+c_{2} \psi \\
& c_{3} \phi \psi-c_{4} \psi^{2}=c_{1} c_{3} \phi^{2}-c_{1} c_{4} \phi \psi+c_{2} \phi
\end{aligned}
$$

Subtracting Equation (6) from Equation (5) gives

$$
c_{4}\left(\psi^{2}-\phi^{2}\right)=c_{1} c_{3}\left(\psi^{2}-\phi^{2}\right)+c_{2}(\psi-\phi) .
$$

This can be arranged as follows

$$
(\phi-\psi)\left[\left(c_{1} c_{3}-c_{4}\right)(\phi+\psi)+c_{2}\right]=0 .
$$

Now, if $c_{1} c_{3}>c_{4}$, then $\phi=\psi$. Therefore, Theorem B in [17] guarantees that the point $\bar{x}$ is a global attractor.

Theorem 3. Let $c_{1}>\frac{c_{2} c_{4} s}{\left(c_{3} r-c_{4} s\right)^{2}}$, then the equilibrium point of Equation (1) is a global attractor if $c_{3}<c_{4}$ and $c_{1}<1$.

Proof. The proof can be accomplished in a similar way to the previous one. Thus, it is omitted.

\section{Periodicity of the solution}

Equation (1) has no prime period two solutions. This property will be proved in the following theorem. 
Theorem 4. The Equation (1) has no prime period two solutions.

Proof. Assume that the Equation (1) has a prime period two solution given as follows

$$
\ldots, t, \tau, t, \tau, \ldots,
$$

with $t \neq \tau$. From Equation (1), one can observe that

$$
\begin{aligned}
t & =c_{1} t+\frac{c_{2} t}{c_{3} t-c_{4} t} \\
\tau & =c_{1} \tau+\frac{c_{2} \tau}{c_{3} \tau-c_{4} \tau} .
\end{aligned}
$$

Hence,

$$
\begin{aligned}
\left(1-c_{1}\right) t & =\frac{c_{2}}{c_{3}-c_{4}} \\
\left(1-c_{1}\right) \tau & =\frac{c_{2}}{c_{3}-c_{4}}
\end{aligned}
$$

This implies that $t=\tau$, which contradicts our assumption.

\section{Special case of Equation (1)}

This section is devoted to present the exact solution of the following recursive equation

$$
x_{n+1}=x_{n-3}+\frac{x_{n-3}}{x_{n-3}-x_{n-7}}, \quad n=0,1, \ldots
$$

Theorem 5. Let $\left\{x_{n}\right\}_{n=-7}^{\infty}$ be a solution of Equation (7) and assume that $x_{-7}=\alpha, x_{-6}=\beta, x_{-5}=\gamma_{,} x_{-4}=$ $\delta, x_{-3}=\epsilon, x_{-2}=\zeta, x_{-1}=\kappa, x_{0}=\omega$. Then, for $n=0,1,2, \ldots$, the solution of Equation (7) is given by the following relations

$$
\begin{aligned}
& x_{8 n-7}=-\frac{[(n-1) \alpha-n \epsilon][\alpha-\epsilon-n]}{\alpha-\epsilon}, \quad x_{8 n-6}=-\frac{[(n-1) \beta-n \zeta][\beta-\zeta-n]}{\beta-\zeta}, \\
& x_{8 n-5}=-\frac{[(n-1) \gamma-n \kappa][\gamma-\kappa-n]}{\gamma-\kappa}, \quad x_{8 n-4}=-\frac{[(n-1) \delta-n \omega][\delta-\omega-n]}{\delta-\omega}, \\
& x_{8 n-3}=-\frac{[n \alpha-(n+1) \epsilon][\alpha-\epsilon-n]}{\alpha-\epsilon}, \quad x_{8 n-2}=-\frac{[n \beta-(n+1) \zeta][\beta-\zeta-n]}{\beta-\zeta}, \\
& x_{8 n-1}=-\frac{[n \gamma-(n+1) \kappa][\gamma-\kappa-n]}{\gamma-\kappa}, \quad x_{8 n}=-\frac{[n \delta-(n+1) \omega][\delta-\omega-n]}{\delta-\omega} .
\end{aligned}
$$

Proof. It can be easily seen that the formulae are true at $n=0$. Next, we assume that $n>0$ and suppose that our solution is correct at $n-1$ as follows

$$
\begin{aligned}
& x_{8 n-15}=-\frac{[(n-2) \alpha-(n-1) \epsilon][\alpha-\epsilon-n+1]}{\alpha-\epsilon}, \quad x_{8 n-14}=-\frac{[(n-2) \beta-(n-1) \zeta][\beta-\zeta-n+1]}{\beta-\zeta}, \\
& x_{8 n-13}=-\frac{[(n-2) \gamma-(n-1) \kappa][\gamma-\kappa-n+1]}{\gamma-\kappa}, \quad x_{8 n-12}=-\frac{[(n-2) \delta-(n-1) \omega][\delta-\omega-n+1]}{\delta-\omega}, \\
& x_{8 n-11}=-\frac{[(n-1) \alpha-n \epsilon][\alpha-\epsilon-n+1]}{\alpha-\epsilon}, \quad x_{8 n-10}=-\frac{[(n-1) \beta-n \zeta][\beta-\zeta-n+1]}{\beta-\zeta}, \\
& x_{8 n-9}=-\frac{[(n-1) \gamma-n \kappa][\gamma-\kappa-n+1]}{\gamma-\kappa}, \quad x_{8 n-8}=-\frac{[(n-1) \delta-n \omega][\delta-\omega-n+1]}{\delta-\omega} .
\end{aligned}
$$

Now, we prove the first relation. Equation (7) gives

$$
x_{8 n-7}=x_{8 n-11}+\frac{x_{8 n-11}}{x_{8 n-11}-x_{8 n-15}}
$$




$$
\begin{aligned}
& =-\frac{[(n-1) \alpha-n \epsilon][\alpha-\epsilon-n+1]}{\alpha-\epsilon}+\frac{-\frac{[(n-1) \alpha-n \epsilon][\alpha-\epsilon-n+1]}{\alpha-\epsilon}}{-\frac{[(n-1) \alpha-n \epsilon][\alpha-\epsilon-n+1]}{\alpha-\epsilon}+\frac{[(n-2) \alpha-(n-1) \epsilon][\alpha-\epsilon-n+1]}{\alpha-\epsilon}} \\
& =-\frac{[(n-1) \alpha-n \epsilon][\alpha-\epsilon-n+1]}{\alpha-\epsilon}-\frac{[(n-1) \alpha-n \epsilon]}{\epsilon-\alpha} \\
& =-\left[\frac{[(n-1) \alpha-n \epsilon][\alpha-\epsilon-n+1]}{\alpha-\epsilon}-\frac{[(n-1) \alpha-n \epsilon]}{\alpha-\epsilon}\right] \\
& =-\frac{[(n-1) \alpha-n \epsilon][\alpha-\epsilon-n]}{\alpha-\epsilon} .
\end{aligned}
$$

We now prove the second formula. Again, Equation (7) gives

$$
\begin{aligned}
x_{8 n-6} & =x_{8 n-10}+\frac{x_{8 n-10}}{x_{8 n-10}-x_{8 n-14}} \\
& =-\frac{[(n-1) \beta-n \zeta][\beta-\zeta-n+1]}{\beta-\zeta}+\frac{-\frac{[(n-1) \beta-n \zeta][\beta-\zeta-n+1]}{\beta-\zeta}}{-\frac{[(n-1) \beta-n \zeta][\beta-\zeta-n+1]}{\beta-\zeta}+\frac{[(n-2) \beta-(n-1) \zeta][\beta-\zeta-n+1]}{\beta-\zeta}} \\
& =-\left[\frac{[(n-1) \beta-n \zeta][\beta-\zeta-n+1]}{\beta-\zeta}-\frac{[(n-1) \beta-n \zeta]]}{\beta-\zeta}\right] \\
& =-\frac{[(n-1) \beta-n \zeta][\beta-\zeta-n]}{\beta-\zeta} .
\end{aligned}
$$

The proofs of the remaining relations can be achieved in a similar way. Thus, the remaining proofs are omitted.

\section{Numerical examples}

This section will confirm the above-mentioned theoretical analysis by providing some numerical examples.

Example 1. In this example, we confirm the local stability of the equilibrium point under the values $c_{1}=$ $0.5, c_{2}=0.3, c_{3}=10, c_{4}=1, x_{-7}=-0.02, x_{-6}=0.15, x_{-5}=0.05, x_{-4}=-0.2, x_{-3}=0.01, x_{-2}=$ $0.2, x_{-1}=-0.01, x_{0}=0.1$. See Figure 1 .

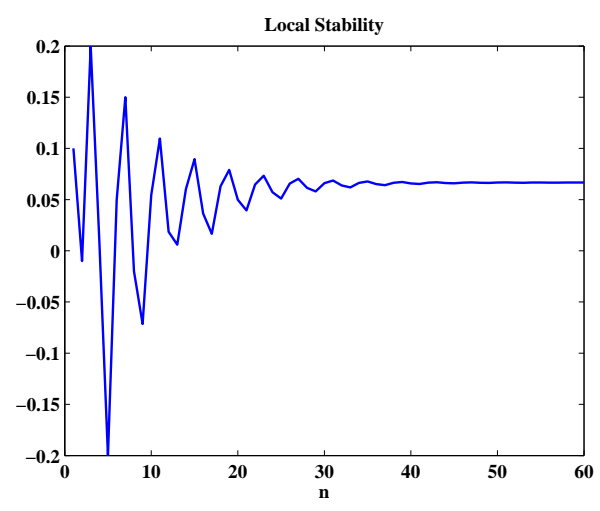

Figure 1. Local Stability of The Equilibrium Point.

Example 2. Figure 2 shows the global stability of the fixed point when we let $c_{1}=0.5, c_{2}=10, c_{3}=3, c_{4}=$ $0.6, x_{-7}=12, x_{-6}=-1, x_{-5}=1, x_{-4}=7, x_{-3}=-9, x_{-2}=10, x_{-1}=-8, x_{0}=11$.

Example 3. In Figure 3, we plot the global stability under the conditions given in Theorem 3 . Here, we consider the values $c_{1}=0.5, c_{2}=0.025, c_{3}=5, c_{4}=6, x_{-7}=1, x_{-6}=2, x_{-5}=-2, x_{-4}=4, x_{-3}=-7, x_{-2}=$ $8, x_{-1}=-10, x_{0}=10$. 


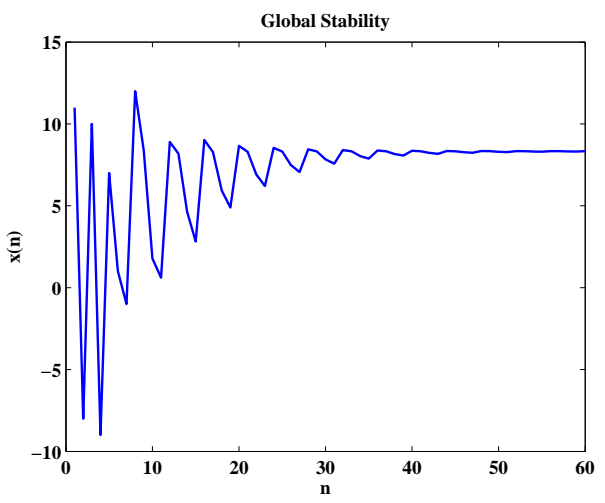

Figure 2. Global Stability of The Equilibrium Point.

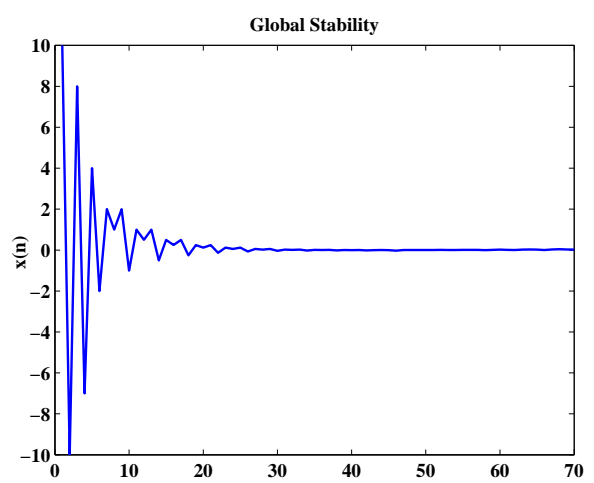

Figure 3. Global Stability of The Equilibrium Point.

Example 4. The behaviour of the solution of Equation (7) is plotted in Figure 4 according to the following values: $c_{1}=0.5, c_{2}=0.025, c_{3}=5, c_{4}=6, x_{-7}=1, x_{-6}=2, x_{-5}=-2, x_{-4}=4, x_{-3}=-7, x_{-2}=$ $8, x_{-1}=-10, x_{0}=10$.

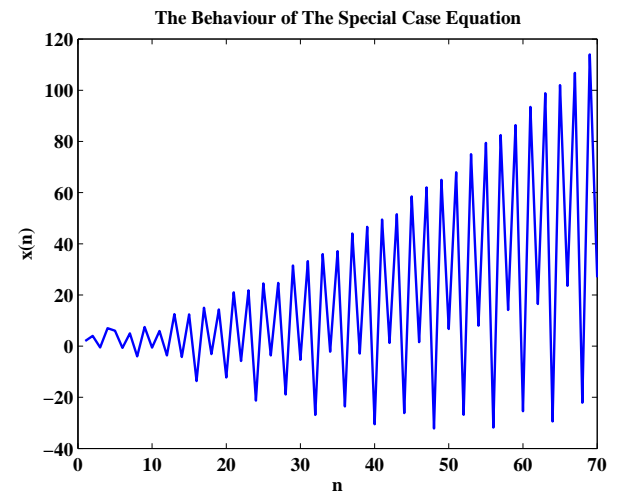

Figure 4. Solution of The Special Case Equation.

\section{Conclusion}

This paper investigated the qualitative behaviour of Equation (1) and shown some numerical examples for these properties. For instance, Theorem 1 presents the condition under which the fixed point of Equation (1) is locally asymptotically stable. Moreover, Theorem 2 provides a simple condition under which the equilibrium point is a global attractor, while Theorem 3 proves the global stability if $c_{3}<c_{4}$ and $c_{1}<1$, hold. In Section 4 , it has been proved that Equation (1) has no a prime period two solution. Theorem 5 introduces the exact 
solution of the Equation (7). Finally, Section 6 has been added to confirm all analytical work discussed in the previous sections.

Author Contributions: All authors contributed equally to the writing of this paper. All authors read and approved the final manuscript.

Conflicts of Interest: “The authors declare no conflict of interest."

\section{References}

[1] Abdelrahman, M. A. E., Chatzarakis, G. E., Tongxing, L., \& Moaaz, O. (2018). On the difference equation $x_{n+1}=$ $a x_{n-l}+b x_{n-k}+f\left(x_{n-l}, x_{n-k}\right)$. Advances in Difference Equations, 2018(1), 1-14.

[2] Almatrafi, M. B., Elsayed, E. M., \& Alzahrani, F. (2018). Qualitative behavior of two rational difference equations. Fundamental Journal of Mathematics and Applications, 1(2), 194-204.

[3] El-Moneam, M. A., \& Zayed, E. M. E. (2014). Dynamics of the rational difference equation. Information Sciences Letters, $3(2), 45-53$.

[4] Garić-Demirović, M., Nurkanović, M., \& Nurkanović, Z. (2017), Stability, periodicity and Neimark-Sacker bifurcation of certain homogeneous fractional difference equations. International Journal of Difference Equations, 12(1), 27-53.

[5] Ghazel, M., Elsayed, E. M., Matouk, A. E., \& Mousallam, A. M. (2017). Investigating dynamical behaviors of the difference dquation $x_{n+1}=C x_{n-5} /\left(A+B x_{n-2} x_{n-5}\right)$. Journal of Nonlinear Sciences and Applications, 10, 4662-4679.

[6] Khaliq, A., \& Hassan, S. S. (2018). Dynamics of a rational difference equation $x_{n+1}=a x_{n}+\left(\alpha+\beta x_{n-k}\right) /\left(A+B x_{n-k}\right)$. International Journal of Advances in Mathematics, 2018(1), 159-179.

[7] Elabbasy, E. M., El-Metwally, H., \& Elsayed, E. M. (2006). On the difference equation $x_{n+1}=a x_{n}-\left(b x_{n}\right) /\left(c x_{n}-\right.$ $\left.d x_{n-1}\right)$. Advances in difference Equations, 2006(1), 1-10.

[8] Almatrafi, M. B., Elsayed, E. M. (2018). Solutions and formulae for some systems of difference equations. MathLAB Journal, 1(3), 356-369.

[9] Almatrafi, M. B., Elsayed, E. M., \& Alzahrani, F. (2019). Qualitative behavior of a quadratic second-order rational difference equation. International Journal of Advances in Mathematics, 2019(1), 1-14.

[10] Belhannache, F., Touafek, N., \& Abo-zeid, R. (2016). On a higher-order rational difference equation. Journal of Applied Mathematics \& Informatics, 34(5-6),369-382.

[11] Kostrov, Y., \& Kudlak, Z. (2016). On a second-order rational difference equation with a quadratic term. International Journal of Difference Equations, 11(2), 179-202.

[12] Liu, K., Li, P., Han, F., \& Zhong, W. (2018). Global dynamics of nonlinear difference equation $x_{n+1}=A+$ $x_{n} / x_{n-1} x_{n-2}$. Journal of Computational Analysis and Applications, 24(6), 1125-1132.

[13] Moranjkić, S., \& Nurkanović, Z. (2017). Local and global dynamics of certain second-order rational difference equations containing quadratic terms. Advances in Dynamical Systems and Applications, 12(2), 123-157.

[14] Saleh, M. \& Aloqeili, M. (2005). On the rational difference equation $y_{n+1}=A+\frac{y_{n-k}}{y_{n}}$. Applied Mathematics and Computation, 171(1), 862-869.

[15] Saleh, M., \& Farhat, A. (2017). Global asymptotic stability of the higher order equation $x_{n+1}=\frac{a x_{n}+b x_{n-k}}{A+B x_{n-k}}$. Journal of Applied Mathematics and Computing, 55(1-2), 135-148.

[16] Elabbasy, E. M., El-Metawally, H., \& Elsayed, E. M. (2008). On the difference equation $x_{n+1}=\left(a x_{n}^{2}+\right.$ $\left.b x_{n-1} x_{n-k}\right) /\left(c x_{n}^{2}+d x_{n-1} x_{n-k}\right)$. Sarajevo Journal of Mathematics, 4(17), 1-10.

[17] Elsayed, E. M., \& Alghamdi, A. (2016). Dynamics and global stability of higher order nonlinear difference equation. Journal of Computational Analysis and Applications, 21(3), 493-503.

(C) 2019 by the authors; licensee PSRP, Lahore, Pakistan. This article is an open access article distributed under the terms and conditions of the Creative Commons Attribution (CC-BY) license (http://creativecommons.org/licenses/by/4.0/). 